\title{
DIAGNÓSTICO DE BEM-ESTAR DE BOVINOS EM DOIS MATADOUROS ESTADUAIS NA REGIÃO SUL DO BRASIL
}

\author{
Janaina da Silva Braga ${ }^{1}$, Melisa Fructuoso Machadoㄹ, Tâmara Duarte Borges'1, \\ Marister de Souza' ${ }^{1}$, Ana Paula de Oliveira Souza ${ }^{1}$, Carla Forte Maiolino Molento ${ }^{1}$ \\ 1 UFPR \\ Correspondência: Janaina da Silva Braga: janainasbraga@yahoo.com.br
}

RESUMO: O objetivo desse trabalho foi diagnosticar o grau de bem-estar de bovinos em dois matadouros de inspeção estadual no estado de Santa Catarina por meio da identificação da incidência de pontos críticos. Planilhas utilizadas para auditoria interna de bem-estar animal em matadouros foram adaptadas. Durante a condução foram observados 156 animais e para a avaliação dos hematomas 128 carcaças. Dos 156 animais, $104(66,6 \%)$ receberam choques elétricos, $38(24,3 \%)$ escorregaram, nove $(5,7 \%)$ caíram e $20(12,8 \%)$ vocalizaram. Para a insensibilização dos animais foi utilizada pistola com dardo cativo penetrante, por vezes adicionado de golpes de marreta no matadouro A e de choupa nos matadouros A e B. Observou-se que apenas $11(7,0 \%)$ bovinos apresentaram indicadores de inconsciência. Apenas três (1,9\%) animais foram sangrados até no máximo um minuto após a insensibilização. Foram quantificadas 294 lesões, indicando uma média de mais de duas lesões por bovino. Conclui-se que as instalações apresentaram pontos críticos e que o impacto dos procedimentos de insensibilização sobre o grau de bem-estar dos animais estudados foi severo, necessitando melhorias para atendimento tanto de questões de bem-estar animal como regulamentares. A adoção de manejo humanitário e o monitoramento dos pontos críticos de bem-estar podem promover aumento de rendimentos e avanço ético para todos os envolvidos.

Palavras-chave: abate humanitário; hematomas; insensibilização; matadouro; pontos críticos

\section{CATTLE WELFARE ASSESSMENT IN TWO STATE REGULATED ABATTOIRS IN SOUTHERN BRAZIL}

\begin{abstract}
The objective of this work was to assess the welfare of cattle in two State regulated abattoirs in Southern Brazil, through the identification of the incidence of critical points. Spreadsheets used for internal auditing of animal welfare in slaughterhouses were adapted. A total of 156 animals were observed during management, and it was observed that $104(66.6 \%)$ animals received electric shocks, nine (5.7\%) fell, 38 (24.3\%) slipped and 20 (12.8\%) vocalized. Regarding the stunning procedure, penetrating captive bolt pistol was used, sometimes associated with blows from mallet in slaughterhouses $A$ and blows from puntilla in slaughterhouses $A$ and $B$. It was observed that only 11 $(7.0 \%)$ animals showed signs of unconsciousness. Only three $(1.9 \%)$ were bled within a maximum period of one minute after stunning. For the evaluation of bruises were observed 128 carcasses. A total of 294 lesions were quantified, indicating an average of more than two lesions per animal. It is concluded that slaughter plants presented critical points and that there was a severe impact of stunning methods on animal welfare. The adoption of humane handling and monitoring of critical welfare points with effective improvement efforts may increase profits and promote ethical progress for all involved.
\end{abstract}

Key Words: bruises; critical point; humane slaughter; slaughterhouse; stunning 


\section{INTRODUÇÃO}

O bem-estar dos animais é uma questão emergente nas sociedades, que tem se preocupado com as condições nas quais os animais são criados, transportados e abatidos e cada vez mais exige a minimização do sofrimento dos animais (SENG; LAPORTE, 2005). Essa atenção relacionada ao sofrimento surge a partir do reconhecimento de que animais vertebrados são sencientes, ou seja, apresentam a capacidade de ter consciência de si mesmos e de suas interações com 0 meio ambiente, possuindo habilidade para experimentar estados de prazer, como a felicidade e estados aversivos, como a dor, o sofrimento e a tristeza (LOW et al., 2012).

Essa preocupação pode ser vista por meio do desconforto da sociedade brasileira quando casos de maus tratos a animais de produção são denunciados pela mídia, como pode ser verificado na reportagem sobre as condições de abate em estabelecimentos municipais e estaduais no Brasil, resultando em instauração de processo ético pelo Conselho Federal de Medicina Veterinária e representação junto ao Ministério Público Federal (CFMV, 2013).

$\begin{array}{ccc}\begin{array}{c}\text { Porém, } \\ \text { estabelecimentos }\end{array} & \begin{array}{c}\text { abate } \\ \text { estaduais }\end{array} & \text { em }\end{array}$
municipais não apresenta somente problemas de bem-estar animal. De acordo com Felício (2013) pode-se caracterizar o cenário pelo abate de 30 animais/dia, comumente animais de descarte, com instalações deficientes e controle sanitário sistemático precário, economicamente inviável, mas tolerado pelo poder público. A abrangência desses estabelecimentos no Brasil é muito significativa, praticamente todos os municípios apresentam um estabelecimento e segundo o IBGE (2013), o abate de bovinos por ano fiscalizado pelo Serviço de Inspeção
Federal (SIF), Serviço de Inspeção Estadual (SIE) e Serviço de Inspeção Municipal (SIM), representam 75,0\%, $17,2 \%$ e $7,8 \%$, respectivamente. Entretanto esse número pode ser subestimado; pois pode haver 12 milhões de animais abatidos clandestinamente, mantidos ilegalmente perante o Decreto Presidencial $n^{\circ} 5.741$ que estabelece a obrigatoriedade prévia de fiscalização e registro no órgão competente (BRASIL, 2006). Então, com abate anual estimado de 42,5 milhões de cabeças com o desfrute de $20 \%$, a participação dos abates registrados pelo IBGE seria de apenas $53,3 \%$ fiscalizados pelo SIF, e $12,2 \%$ e $5,5 \%$ pelas inspeções estaduais e municipais, respectivamente.

Embora os estabelecimentos estaduais e municipais representem uma parcela significativa do abate anual de bovinos, pesquisas desenvolvidas para entender a realidade desses estabelecimentos em relação ao tratamento humanitário dos animais são escassas. A maioria das pesquisas já desenvolvidas nessa área no Brasil (ALMEIDA, 2005; BARBALHO, 2007; ANDRADE et al., 2008; NEVES, 2008; ANDRADE; COELHO, 2011; BERTOLONI et al., 2012; DUTRA; PEREIRA, 2012), são provenientes de frigoríficos sob Serviço de Inspeção Federal (SIF) do Ministério da Agricultura, Pecuária e Abastecimento (MAPA).

Dessa forma, o objetivo desse trabalho foi diagnosticar o grau de bemestar de bovinos em dois matadouros de inspeção estadual no estado de Santa Catarina, região Sul do Brasil, por meio da identificação da incidência de pontos críticos selecionados de bem-estar animal.

\section{MATERIAL E MÉTODOS}


A coleta de dados ocorreu em dois matadouros estaduais aqui denominados $\mathrm{A}$ e $\mathrm{B}$, ambos de abate exclusivo de bovinos, num total de 156 animais. Para a coleta de dados foram elaboradas planilhas adaptadas das diretrizes do Instituto Americano de Carnes (GRANDIN, 2001) e das diretrizes recomendadas para o manejo animal e guia de auditoria para bovinos, suínos e ovinos (GRANDIN, 2005).

A coleta de dados foi dividida em cinco etapas: avaliações das instalações, do manejo de condução dos bovinos dos currais de espera até o tronco de insensibilização, da eficácia do procedimento de insensibilização, dos indicadores de insensibilização e sangria e da quantidade, localização e coloração dos hematomas nas carcaças bovinas.

A primeira avaliação teve por objetivo caracterizar as condições das instalações especificamente na plataforma e rampa de desembarque, nos currais de espera, no corredor de abate, no banheiro de aspersão, na seringa e no tronco de insensibilização.

$\mathrm{Na}$ segunda avaliação, os comportamentos dos funcionários e dos bovinos registrados foram baseados no protocolo definido por Grandin (2005), registrando-se: o uso de bastão elétrico pelo funcionário, escorregões, quedas e vocalizações apresentadas pelos bovinos. Considerou-se a ocorrência do uso do bastão elétrico cada vez que o bastão tocava o corpo de um bovino durante o manejo. Um escorregão foi registrado cada vez que o animal se desequilibrava, ocorrendo deslocamento involuntário de algum membro. Queda, quando o animal foi ao chão pelo próprio peso ou por desequilíbrio, tocando o piso com qualquer parte do corpo exceto cascos. Qualquer som audível emitido pelo animal no corredor de abate, no banheiro de aspersão, na seringa e no brete foi considerado vocalização. O início do registro dos comportamentos referentes à condução dos bovinos ocorreu no momento da abertura da porteira do curral de espera para a condução dos animais, em lotes. Os lotes foram acompanhados durante todo o percurso, incluindo curral de espera, corredor de abate e banheiro de aspersão. Os lotes foram acompanhados de maneira independente de sexo, categoria e origem dos animais.

A terceira avaliação teve por objetivo avaliar a eficácia do procedimento de insensibilização. Para isso, foram quantificados o número de disparos da pistola de dardo cativo recebido pelos animais e a presença de indicadores de consciência. Esta prática foi descrita como um indicador de bemestar animal em plantas frigoríficas por Grandin (2005). Foram medidos com o uso de um cronômetro o momento do primeiro disparo da pistola pneumática de dardo cativo penetrante e o momento do corte dos grandes vasos do pescoço do animal, para posterior cálculo do tempo entre a insensibilização e a sangria.

A quarta avaliação teve por objetivo avaliar os indicadores de inconsciência dos bovinos logo após os animais serem içados na canaleta de sangria. A avaliação da insensibilização inadequada foi realizada de acordo com a presença de um ou mais dos seguintes indicadores: vocalizações, como qualquer som audível emitido pelo bovino; respiração rítmica, observada pela presença de movimentos respiratórios rítmicos, como movimentos da narina e do flanco do bovino; movimentos oculares e ciliares, observados pelo movimento lateral dos olhos e fechamento da pálpebra do bovino em resposta a um toque com as pontas dos dedos; reflexo de correção de postura, como tentativas do bovino de levantar o pescoço para se colocar 
em posição de pé; ausência de protrusão de língua, observada pela retenção da língua da cavidade oral; e cauda tensa, observada pela ausência de relaxamento ou movimentação da cauda. Foram considerados conscientes os animais que apresentaram um ou mais destes indicadores.

$\mathrm{Na}$ quinta avaliação, a caracterização dos hematomas das carcaças foi baseada no Sistema Australiano de Avaliação de Contusões nas Carcaças (ANDERSON e HORDER, 1979). Os hematomas foram observados de forma visual durante a inspeção interna e externa das partes caudal e cranial das carcaças. Os hematomas foram classificados de acordo com a coloração vermelha ou amarela. Os de coloração vermelha ou vermelha escura foram considerados decorrentes do transporte ou do manejo pré-abate; e o de coloração amarela foi classificado como anterior ao transporte ou ao manejo pré-abate, de acordo com Grandin (2004). A resenha para registro das ocorrências de hematomas consistiu de carcaças esquemáticas subdivididas em três zonas, Zona 1 (membro pélvico), Zona 2 (ponta de agulha), Zona 3 (membro torácico). Cada lesão foi marcada de acordo com a sua região e coloração.

Para a análise dos resultados utilizou-se a estatística descritiva do Programa Excel 2007.

\section{RESULTADOS E DISCUSSÃO}

Observou-se que no matadouro $A$ há ausência de uma plataforma plana antes da rampa para o de desembarque dos animais no matadouro, a qual de acordo com Grandin (2004) evita a queda de animais. A rampa de desembarque apresentava $32^{\circ}$, e de acordo com Grandin (2007a) as rampas devem ter no máximo $20^{\circ}$, uma vez que rampas muito íngremes podem proporcionar injúrias aos animais, causando sofrimento desnecessário e afetando negativamente o grau de bemestar dos animais. A rampa de desembarque apresentava piso de cimento, sem frisos antiderrapantes, era limitada por uma parede de cimento e a outra formada por tubos galvanizados, com espaço médio de $30 \mathrm{~cm}$ entre eles. De acordo com Grandin (2004), as rampas devem ter as paredes sólidas, pois isso acalma os bovinos e facilita o manejo, uma vez que impede a visão de distrações tais como pessoas e veículos.

Nos currais de espera observou-se a ausência de bebedouros no matadouro $A$; no matadouro $B$ observou-se a presença de cinco currais e apenas um bebedouro que armazenava água em reservatório com limo, grossa camada de sujeira e insetos mortos na superfície da água. Observou-se a presença de animais que tentaram beber a água que estava acumulada no banheiro de imersão, quando conduzidos dos currais ao tronco de insensibilização, no matadouro B. De acordo com Gregory (1998), a presença de sede é um ponto crítico comum durante o transporte préabate. A ausência de bebedouros nos currais está em desacordo com os requisitos aplicáveis aos estabelecimentos de abate descritos na IN003/2000, segundo os quais os animais mantidos nos currais devem ter livre acesso a água limpa e abundante (Brasil, 2000). De acordo com Mellor et al. (2009), os estímulos sensoriais e outros estímulos neurais associados à privação de água junto com estímulos cognitivos adicionais são processados e expressos sob a forma de sentimento subjetivo consciente nos animais. Quanto maior a intensidade dos sentimentos subjetivos negativos, maior será o comprometimento do grau de bem-estar dos animais, podendo levar ao sofrimento em casos extremos. 
Observou-se no matadouro A que o corredor de abate era retilíneo, limitado por paredes formadas por estacas de madeira com espaço entre elas de $15 \mathrm{~cm}$. Corredores suavemente curvilíneos e com paredes sólidas facilitam o manejo uma vez que previnem que o bovino veja para onde está indo, aproveitam a tendência natural do animal de circular em volta do manejador e o comportamento natural dos bovinos de desejarem voltar para onde vieram (GRANDIN, 2004).

Nos matadouros A e B observouse que os troncos de insensibilização continham um portão sólido de metal do tipo guilhotina, parede de cimento e uma porta de metal com movimento basculante lateral móvel que após o procedimento de insensibilização era aberta e ejetava os animais para a área de sangria. Não havia a presença de nenhum equipamento que contivesse 0 animal dentro do tronco. $O$ tronco de insensibilização não se mostrou eficaz na contenção dos animais, permitindo que 0 animal se movimentasse se virasse e tentasse fugir do disparo, dificultando a insensibilização. De acordo com Grandin (2010b), o tronco de insensibilização deve ter dimensões adequadas para impedir que 0 animal se vire, com isso se restringe ao máximo os movimentos dos animais, aumentando a eficiência de insensibilização ao primeiro disparo da pistola.

Dos 156 animais avaliados nos matadouros $A$ e $B$, observou-se que 0 uso de choque elétrico para a condução dos bovinos dos currais de espera até a seringa foi excessivo (Figura 1) em ambos os matadouros, semelhante ao encontrado por Braga (2010a). Em 104 animais $(66,6 \%)$ foi utilizado choque elétrico, sendo que destes 31 (19,8\%) receberam choques elétricos com duração superior a dez segundos, três
$(1,9 \%)$ receberam choques na narina $e$ um $(0,6 \%)$ no ânus.

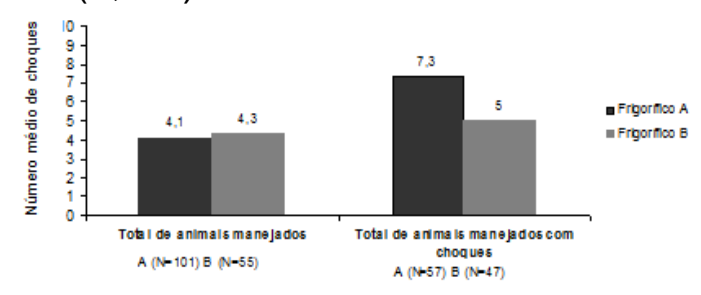

Figura 1 - Número médio de choques elétricos durante a condução de bovinos nos matadouros A e B. Valores sobre o tdal de animais manejados e sobre total de animais manejados com choque, ambos sob Serviço de Inspeção Estadual no estado de Santa Catarina, região Sul do Brasil, em 2010.

A utilização de bastão elétrico para condução dos bovinos nesses matadouros está em desacordo com a legislação brasileira, que afirma que os dispositivos de descargas elétricas só deverão ser utilizados quando os animais se recusarem a mover desde que essa descarga não dure mais que dois segundos e que somente deverão ser aplicados nos membros (BRASIL, 2000). De acordo com Grandin (2000), considera-se o uso de choques elétricos nesses matadouros um grave problema de bem-estar animal, pois são usados em mais de $25 \%$ dos animais. Os choques não devem ser o principal instrumento para condução dos animais e sempre que possível devem ser substituídos por instrumentos alternativos como a bandeira (GRANDIN, 2010c). É de extrema importância que o manejador seja treinado sobre alguns conceitos básicos como o de zona de fuga e ponto de equilíbrio dos bovinos (GRANDIN, 2010d), para que a bandeira seja utilizada para uma condução eficaz.

Com relação ao comportamento dos bovinos, pode-se observar que 38 $(24,3 \%)$ escorregaram e nove $(5,7 \%)$ caíram durante o manejo de condução. Esses porcentuais estão acima das recomendações de Grandin (2001), que estabelecem um máximo de $3 \%$ de escorregões e $1 \%$ de quedas. Esses porcentuais de quedas e escorregões podem ser atribuídos às deficiências nas instalações e principalmente ao 
manejo inadequado desses animais, com a provável associação de ambos. Com relação à vocalização, 20 (12,8\%) vocalizaram durante a condução até o tronco de insensibilização. De acordo com Grandin (2001), os resultados são inaceitáveis quando mais de $3 \%$ vocalizam. As vocalizações são indicativas da presença de dor ou medo no manejo e estão associadas com a ocorrência de eventos aversivos tais como o uso excessivo de choques, falha na insensibilização, escorregões e quedas durante a condução (GRANDIN, 1998), observados nas condições desses matadouros.

Observou-se no matadouro $A$ que oito $(5,1 \%)$ animais foram conduzidos ao tronco de insensibilização em duplas. De acordo com Grandin (2010b) cada animal deve ser conduzido ao tronco de insensibilização sozinho, para evitar o pisoteio de um animal pelo outro.

A variedade na quantidade de disparos da pistola pode ser observada na Tabela 1. Observa-se que apenas 56 $(35,8 \%)$ animais do matadouro $A$ receberam disparos da pistola. Observou-se que 37 (23,7\%) dos animais receberam três ou mais disparos, resultado superior ao encontrado por Braga (2010a e 2010b).

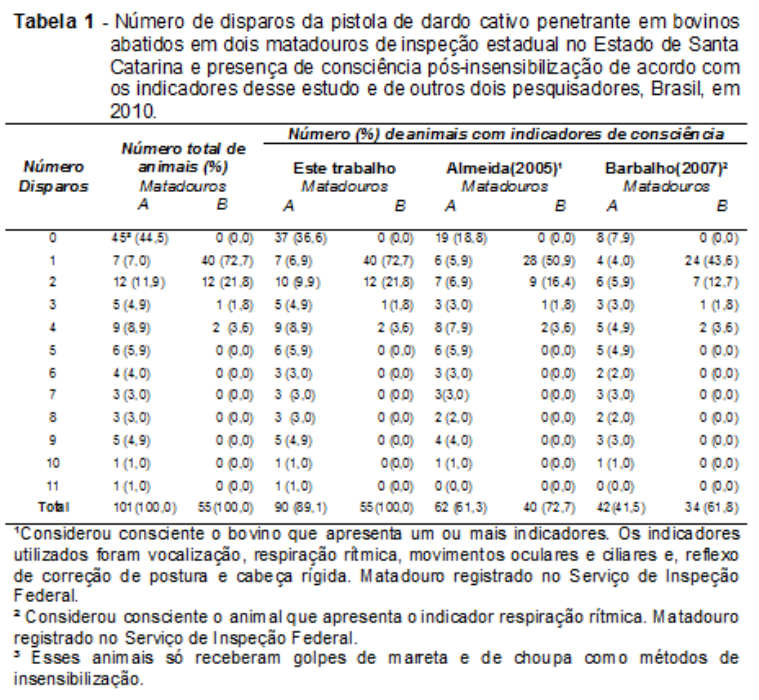

Observa-se na Tabela 1 que apenas $47(30,1 \%)$ bovinos receberam um disparo da pistola e destes, 47
$(100,0 \%)$ apresentaram sinais de consciência após o disparo. Grandin (2001) considera aceitável quando 95\% e excelente quando 99 a $100 \%$ dos bovinos são insensibilizados no primeiro disparo. De acordo com os resultados obtidos nesse estudo observa-se um nível alto de ineficácia de insensibilização ao primeiro disparo da pistola nos dois matadouros estudados.

As variações no número de disparos da pistola podem ser atribuídas à manutenção deficiente, como a ausência de limpeza e lubrificação diária da pistola, que no matadouro $A$ apresentou a retenção do pistão após o primeiro disparo em seis $(3,8 \%)$ bovinos. Para desemperrar o pistão, o funcionário da insensibilização utilizou uma faca. Observou-se que em dois $(1,2 \%)$ animais o pistão ficou retido no crânio do animal. Observou-se a ausência de capacitação do funcionário com relação à posição frontal para o disparo e à manutenção da pistola. Observou-se no matadouro $B$ que três $(1,9 \%)$ animais receberam disparos da pistola da região do chifre e quatro $(2,5 \%)$ na região da órbita ocular. Grandin (2002) afirmou que o problema mais comum de bem-estar animal durante a insensibilização é a falta de manutenção da pistola. A pistola é uma ferramenta de precisão e deve ser revisada regularmente. Ainda, de acordo com Gallo et al. (2003), a utilização de um tronco de insensibilização com um contensor de movimento de cabeça, um compressor exclusivo de ar para a pistola e a capacitação dos funcionários apresenta um progresso evidente para o bem-estar dos animais, aumentando de $72,5 \%$ para $97,8 \%$ dos bovinos que apresentaram colapso imediato após o primeiro disparo. É provável que tais propostas se ajustem bem à situação dos dois matadouros estudados.

Observou-se que golpes de marreta e de choupa foram disparados contra o bovino dentro do tronco de 
insensibilização, na área de sangria ou enquanto os animais estavam içados na canaleta de sangria. Os golpes de choupa foram caracterizados pela introdução de uma lança de ferro pontiaguda ou de uma faca de lâmina única no forame oval no espaço atlantooccipital dos bovinos, no matadouro A. No matadouro $B$, observou-se somente os golpes com a faca de lâmina única. $A$ lança pontiaguda era utilizada dentro do tronco de insensibilização enquanto que a faca de lâmina única era utilizada na área de sangria.

Os golpes de marreta foram utilizados em 45 (28,8\%) animais como o principal método de insensibilização dentro do tronco e em 34 (21,7\%) como método adicional ao uso da pistola no matadouro $A$. No matadouro $B$ não se observou a presença de golpes de marretas disparados contra os animais. No matadouro $B$ os animais não recebiam nenhum método adicional após a ineficiência do método de eleição de insensibilização do matadouro, a pistola. Foram disparados 260 golpes de marreta contra 79 bovinos, uma média de 3,2 golpes por bovino (Figura 2). De acordo com Almeida (2005), o abate com marreta depende da habilidade e força física do operador, o que pode ser observado pelas diferenças no número de golpes de marretas nos bovinos (Figura 2).

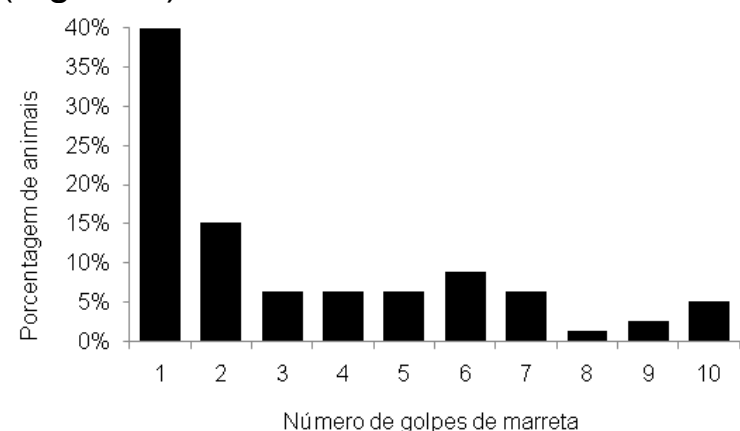

Figura 2 - Número de golpes de marretas disparados contra os bovinos dentro do tronco de insensibilização ou na área de sangria no matadouro $\mathrm{A}$ sob serviço de inspeção estadual no estado de Santa Catarina, região Sul do Brasil, em 2010 .

Os golpes de choupa foram disparados contra 11 (7,0\%) animais, adicionalmente ao uso da pistola ou da marreta. Desses, um $(0,6 \%)$ recebeu oito golpes de choupa, dois $(1,2 \%)$ receberam seis golpes, dois $(1,2 \%)$ três golpes e seis $(3,8 \%)$ um golpe. Essa repetição dos golpes de choupa está diretamente relacionada com a dificuldade em se seccionar a medula totalmente, o que é muito difícil de ser conseguido na prática, sendo que cada repetição do golpe provoca a experiência de dor extrema nos animais. Nesse estudo, adicionalmente à dor experimentada pelos bovinos ao receber os golpes de choupa, houve a dor advinda dos disparos da pistola recebidos e dos golpes de marreta adicionais. Todos estes fatores interferem negativamente no grau de bem-estar dos bovinos. A situação é agravada pela constatação de que, após a utilização da pistola adicionada de marreta e de golpes de choupa, nove $(5,7 \%)$ animais apresentavam um ou mais indicadores de consciência. $O$ método choupa é proibido pela Organização Mundial de Saúde Animal (OIE, 2009), mas de acordo com Nhat (2006), Osborne (2009) e Braga (2010b), ainda é usado em alguns países em desenvolvimento, de forma coerente com os resultados encontrados. Adicionalmente, no Brasil os golpes de marreta e de choupas para a insensibilização dos bovinos não são considerados métodos humanitários de insensibilização de bovinos (Brasil, 2000), ao contrário da pistola de dardo cativo. A presença de golpes de marreta e de choupa, observada nesse estudo, para a insensibilização de bovinos não tem sido descritas nas pesquisas sobre bem-estar de bovinos em matadouros brasileiros sob serviço de inspeção federal (ALMEIDA, 2005; BARBALHO, 2007; NEVES, 2008), sendo descrita em matadouros sob serviço de inspeção municipal (BRAGA 2010a e 2010b).

Dos 156 bovinos observados, 68 $(43,5 \%)$ apresentaram as posições dos 
membros indicativas de insensibilização eficiente. Observou-se que apenas 11 $(7,0 \%)$ apresentaram sinais de inconsciência após os procedimentos de insensibilização. Com relação à presença dos indicadores de consciência, 110 (70,5\%) apresentaram a cauda tensa, 102 (65,3\%) ausência de protrusão de língua, 95 (60,8\%) correção de postura, 81 (51,9\%) respiração rítmica, $25 \quad(16,0 \%)$ movimentos oculares e três $(1,9 \%)$ vocalização. De acordo com Grandin (2001) considera-se aceitável quando um em 500 animais, em abatedouros de grande fluxo, apresenta indicadores de consciência. A presença de consciência, de acordo com Velarde et al. (2003), influencia negativamente a qualidade da carne e o grau de bem-estar dos animais, uma vez que representa uma experiência de dor e distresse para 0 bovino (VIOLA et al., 2004). Existe uma grande divergência de indicadores utilizados para se avaliar a inconsciência em bovinos após a insensibilização. Enriquecendo esse cenário, pode-se incluir o estudo de Gouveia et al., (2009) que sugeriram que os melhores indicadores são a ausência de tônus muscular nas orelhas, presença de espasmos musculares, ausência de respiração rítmica e vocalização. Grandin (2010a) sugere a necessidade de mais pesquisas sobre a relação entre os sinais clínicos que podem ser observados nos matadouros e as avaliações de insensibilidade no laboratório. Gouveia et al., (2009) indica a necessidade de consideração de mais de um indicador de consciência nos bovinos para aumentar a probabilidade de se indentificar um animal consciente após a insensibilização. Pela perspectiva do bem-estar dos animais, após a ocorrência desses indicadores é justificável uma reinsensibilização com o objetivo de evitar o sofrimento desnecessário dos animais.
Pelo âmbito das divergências entre os pesquisadores, observa-se que não existe um único indicador que assegure a presença de consciência do bovino. Então, faz-se necessário a utilização de alguns indicadores que potencialmente possam assegurar que o bovino está inconsciente e será abatido com o mínimo sofrimento possível. Nesse estudo pode-se observar a alta ocorrência de mais de um indicador de consciência por animal.

Apenas três (1,9\%) animais foram sangrados até no máximo um minuto após a insensibilização, fato em desacordo com a legislação brasileira vigente (Brasil, 2000), sendo que o tempo médio entre a insensibilização e a sangria foi de 4'45" \pm 3'33". Observouse no matadouro $A$ que o mesmo funcionário que insensibilizava os animais também auxiliava na sangria. Tal tempo superior entre a insensibilização e a sangria encontrado nos matadouros $A$ e $B$ provavelmente ocorre devido às mesmas causas relatadas por Gallo et al., (2003): desconhecimento da importância entre o tempo da insensibilização e a sangria por parte dos funcionários e falta de coordenação entre ambos. Tais fatores podem ser corrigidos com capacitação e supervisão dos funcionários pelos gestores. Para Gregory e Shaw (2000), do ponto de vista do bem-estar dos bovinos, quando o procedimento de insensibilização é eficaz a preocupação com o tempo entre a insensibilização e a sangria é irrelevante, sendo importante do ponto de vista da qualidade da carne. Entretanto, nas condições desse estudo, nas quais se observou que 145 (92,9\%) bovinos apresentaram indicadores de consciência pós-insensibilização, a preocupação com o tempo é de extrema importância, sendo mais um dos principais pontos críticos de bem-estar de bovinos nesses matadouros. 
Para a avaliação dos hematomas foram observadas 128 carcaças. Foram quantificadas 294 lesões (Figura 3) nos 128 bovinos, indicando uma média de mais de duas lesões por bovino, com destaque para a quantidade de hematomas recentes.

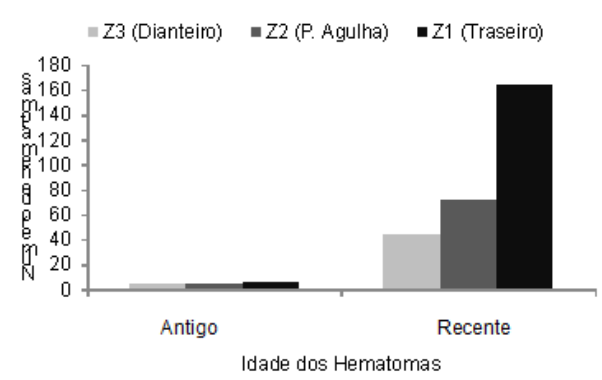

Figura 3 - Número de hematomas por local de ocorrência classificados err anteriores (antigo) e decorrentes (recente) do transporte e manejo préabate em 128 carcaças bovinas nos matadouros A e B, sob Serviço de Inspeção Estadual no Estado de Santa Catarina, região Sul do Brasil,
em 2010 .

Observou-se que apenas 37 $(28,9 \%)$ das carcaças não apresentaram hematomas, valor superior ao encontrado por Braga (2010a e 2010b) em matadouros municipais, de apenas $7,1 \%$ e $9,0 \%$ de carcaças sem nenhum hematoma. Observa-se na Figura 3 que a maior prevalência dos hematomas foi nos quartos traseiro e na ponta de agulha do animal, semelhante ao encontrado por Renner (2005) e Civeira et al., (2006).

De acordo com Jarvis e Cockran (1994) os hematomas geram perdas diretas por meio da desfiguração de cortes musculares e da depreciação das carcaças e indiretas por meio do estresse dos animais, além da perda de qualidade de carcaça, pois as regiões afetadas devem ser retiradas antes da pesagem da mesma (ROÇA, 2002). Durantes as observações para a coleta de dados, apenas uma lesão com $15 \mathrm{~cm}$ de diâmetro foi retirada da carcaça. Pode-se afirmar que as perdas diretas devido a hematomas nesses matadouros foram mínimas ou nulas. De acordo com a FAO e HSI (2001), a carne com hematomas não é aceita pelo consumidor, não pode ser utilizada em carnes processadas e se decompõe rapidamente devido ao sangue ser um meio ideal para o crescimento de bactérias deteriorantes. Além das perdas econômicas, as injúrias que promovem os hematomas são dolorosas (GREGORY, 1996). Desta forma, hematomas representam um importante ponto crítico de sofrimento animal, interferindo diretamente no grau de bem-estar dos animais no momento do abate.

De acordo com a FAO (2008), as áreas relacionadas ao bem-estar dos animais são extremamente diversificadas. Apesar dessa diversificação, a contenção, o manejo e os procedimentos de insensibilização dos animais são áreas problemáticas em diferentes regiões. Os resultados encontrados nesse estudo indicam a necessidade prioritária de estudos sobre bem-estar de bovinos em matadouros brasileiros, com especial atenção àqueles de menor porte e de inspeção regional. De acordo com os critérios estabelecidos por Grandin (2007b) para auditorias em bem-estar de bovinos, os dois matadouros em estudo necessitam de ações corretivas para melhorar o grau de bem-estar animal. No Brasil há a preocupação com o abate de bovinos devido à grande quantidade de animais envolvidos. $O$ atendimento das exigências da sociedade, que deseja a proteção e minimização do sofrimento dos animais no momento do abate, se faz necessário em matadouros de todas as autarquias no cenário brasileiro.

\section{CONCLUSÃO}

As instalações apresentaram pontos críticos para o bem-estar animal e o grau de bem-estar dos bovinos foi baixo durante a condução dos currais de espera até o tronco de insensibilização, principalmente pelo uso de choques elétricos excessivos. O impacto dos procedimentos de insensibilização sobre o grau de bem-estar dos animais 
estudados foi severo, necessitando melhorias para atendimento tanto de questões de bem-estar animal como regulamentares. A adoção de manejo humanitário e o monitoramento dos pontos críticos de bem-estar podem promover aumento de rendimento para o estabelecimento de abate e avanço ético para todos os envolvidos.

\section{AGRADECIMENTOS}

Agradecemos a todos os funcionários dos matadouros estaduais pela colaboração para o desenvolvimento desse estudo.

\section{REFERÊNCIAS}

\section{ALMEIDA, L.A.M. Manejo no pré-abate de bovinos: aspectos comportamentais e perdas econômicas por contusões. $75 f$. Dissertação (Mestrado em Medicina Veterinária Preventiva) - Faculdade de Ciências Agrárias e Veterinária/Universidade Estadual Paulista "Julio de Mesquita Filho", Jaboticabal, 2005.}

ANDERSON, B.; HORDER, J. C. The Australian carcass bruises scoring system. Queensland Agricultural Journal, v.105, p.281-287, 1979.

ANDRADE, N.E.; SILVA, R.A.M.S.; ROÇA, R.O et al. Ocorrência de lesões em carcaças de bovinos de corte no Pantanal em função do transporte. Ciência Rural, v.38, n.7, p.19911996, 2008.

ANDRADE, J.; COELHO, H. E. Ocorrência de contusões em carcaças bovinas e suas perdas econômicas. Cadernos de Pós-Graduação da FAZU, v.1, 2011.

BARBALHO, P.C. Avaliação de programas de treinamento em manejo racional de bovinos em abatedouros para melhoria do bem-estar animal. 2007. Jaboticabal, 70f. Dissertação (Mestrado em Zootecnia) - Faculdade de Ciências Agrárias e Veterinária/Universidade Estadual Paulista "Julio de Mesquita Filho".

BERTOLONI, W. et al. Bem-estar e taxa de hematomas de bovinos transportados em diferentes distâncias e modelos de carroceria no estado do Mato Grosso-Brasil. Revista

Brasileira de Saúde e Produção Animal, v.13, n.3, 2012.

BRAGA, J.S. Diagnóstico do grau de bemestar de bovinos em abatedouros municipais e estaduais no Brasil. Capítulo II. Bem-estar de bovinos de corte em frigorífico municipal na região Sudeste do Brasil. 2010. Curitiba, $126 f$. Dissertação (Mestrado em Ciências Veterinárias) - Setor de Ciências Agrárias, Universidade Federal do Paraná, 2010a.

BRAGA, J.S. Diagnóstico do grau de bemestar de bovinos em abatedouros municipais e estaduais no Brasil. Capítulo III. Bem-estar de bovinos em abatedouro sob serviço de inspeção municipal na região Sul, Brasil._2010. Curitiba, 126f. Dissertação (Mestrado em Ciências Veterinárias) - Setor de Ciências Agrárias, Universidade Federal do Paraná, 2010b.

BRASIL. 2006. Decreto $n^{\circ}$. 5.741 de 30 de março de 2006 Regulamenta os arts. 27-A, 28-A e 29-A da Lei n'8.171, de 17 de janeiro de 1991, organiza o Sistema Unificado de Atenção à Sanidade Agropecuária, e dá outras providências.Diário Oficial [da] República Federativa do Brasil, Rio de Janeiro, RJ. 30 de março de 2006. Disponível em:

<http://www.planalto.gov.br/ccivil_03/_Ato20042006/2006/Decreto/D5741.htm> Acesso em: 25/10/2013.

BRASIL. Instrução Normativa n.03, de 17 de janeiro de 2000. Aprova o regulamento técnico de métodos de insensibilização para o abate humanitário de animais de açougue. Diário Oficial [da] República Federativa do Brasil, Brasília, DF, 18 jan. 2000.

CIVEIRA, M. P.; RENNER, R. M.; VARGAS, R. E. S. et al. Avaliação do bem-estar animal em bovinos abatidos para consumo em frigorífico do Rio Grande do Sul. Veterinária em Foco. v.4, n.1. p.5-11, 2006.

CFMV - CONSELHO FEDERAL DE MEDICINA VETERINÁRIA. Nota pública. Disponível em: <http://www.cfmv.org.br/portal/destaque.php?co $d=1157$ > . Acesso em 01 ago. 2013.

DUTRA, S.; PEREIRA, F. B. Diagnóstico de situação das práticas de manejo sanitário em sistemas de produção de bovinos de corte. Veterinária e Zootecnia, v.19, n.4, p.522-530, 2012.

FAO - Food and Agriculture Organization of the United Nations - Regional Office for Asia and the Pacific - and Humane Slaughter International (HSI). Guidelines for Humane Handling, Transport and Slaughter of Livestock. 2001. Disponível em < http://www.fao.org/docrep/003/x6909e/x6909e00 .HTM>. Acesso em 22/11/2010.

FAO - Food and Agriculture Organization of the United Nations. Capacitação para implementar 
boas práticas de bem-estar animal. 2008 Disponível em:

<http://ftp.fao.org/docrep/fao/012/i0483pt/i0483pt 00.pdf>. Acesso em: 22/11/2010.

FELICIO, P. 2013. A inviabilidade técnica dos pequenos matadouros. Disponível em: http://www.beefpoint.com.br/cadeiaprodutiva/espaco-aberto/a-inviabilidade-tecnicados-pequenos-matadouros/ Acesso em: 25/10/2013.

GALLO, C.; TEUBER, C.; CARTES, M. et al. Mejoras en la insensibilización de bovinos con pistola pneumática neumática de proyectil retenido tras cambios de equipamiento y capacitación del personal. Archivos de Medicina Veterinaria, v.35, n.2, p.159-170, 2003.

GRANDIN, T. The feasibility of using vocalization scoring as an indicator of poor welfare during cattle slaughter. Applied Animal Behaviour Science. v.56, p.121-128, 1998.

GRANDIN, T. Effect of animal welfare audits of slaughter plants by a major fast food company on the cattle handling and stunning practices. Journal of American Veterinary Medical Association, v.216, n.8, p.848-851, 2000.

GRANDIN, T. [2001]. Cattle Slaughter Audit Form Based on American Meat Institute Guidelines, Disponível em:

<http://www.grandin.com/cattle.audit.form.html.> Acesso em: 22/11/2010.

GRANDIN, T. Return to sensibility problems after penetrating captive bolt stunning of cattle in commercial beef slaughter plants. Journal of American Veterinary Medical Association. v.221, p.1258-1261, 2002.

GRANDIN, T. Principles for the design of handling facilities and transport systems. BENSON, G. J.; ROLLIN, B.E.(Eds) The Wellbeing of Farm Animals. Iowa: Blackwell Publishing, 2004. p.145-163.

GRANDIN, T. [2005]. Recommended Animal Handling Guidelines and Audit Guide for Cattle, Pigs, and Sheep. Disponível em: <http://www.grandin.com/RecAnimalHandlingGui delines.html> Acesso em 22/11/2010.

GRANDIN, T. Handling Facilities and Restraint of Range Cattle. In: GRANDIN, T. (Ed.). Livestock Handling and Transport. $3^{\text {nd }}$ ed. Oxfordshire: CABI International

Publishing:,2007a. p.93-97.

GRANDIN, T. Effect of Customer Requirements, International Standards and Marketing Structure on the Handling and Transport of Livestock and
Poultry. Livestock Handling and Transport. $3^{\text {nd }}$ ed. Oxfordshire: CABI International

Publishing:,2007b. p.4-5.

GRANDIN, T. Review auditing animal welfare at slaughter plants. Meat Science. v.86, p.56-65, 2010a.

GRANDIN, T. [2010b]. Proper cattle restraint for stunning. Disponível em:

<http://www.grandin.com/humane/restrain.slaug hter.html>. Acesso em: 22/11/2010.

GRANDIN, T. [2010c]. Using Prods and Persuaders Properly. Disponível em: < http://www.grandin.com/behaviour/principles/pro ds.html>. Acesso em 22/11/2010.

GRANDIN, T. [2010d]. Understanding Flight

Zone and Point of Balance to Improve Handling of Cattle, Sheep, and Pigs.

Disponível em:

<http://www.grandin.com/behaviour/principles/fli ght.zone.html> Acesso em: 22/11/2010.

GOUVEIA, K.G.; FERREIRA, P.G.; ROQUE DA COSTA, J.C. et al. Assessment of the efficiency of captive-bolt stunning in cattle and feasibility of associated behavioural signs. Animal Welfare, v.18, p.171-175, 2009.

GREGORY, N. Welfare and hygiene during preslaughter handling. Meat Science, v.43, p.3546, 1996.

GREGORY, N.G. Stunning and slaughter. GREGORY, N.; GRANDIN, T. (Ed.). Animal Welfare and Meat Science. Oxon: CAB International Publishing: 1998. p.223-240.

GREGORY, N.G.; SHAW, F. Penetrating captive bolt stunning and exsanguinations of cattle in abattoirs. Journal of Applied Animal Welfare Science, v.3, n.3, p.215-230, 2000.

IBGE. Instituto Brasileiro de Geografia e Estatística. Rio de Janeiro, RJ. Disponível em: <http://www.sidra.ibge.gov.br/bda/acervo/acervo 2. $a s p ? e=v \& p=A X \& z=t \& 0=24>$ Acesso em 25/10/2013.

JARVIS, A.M.; COCKRAN, M.S. Effects of handling and transport on bruising of sheep sent directly from farms to slaughter. Veterinary Record, v.135, n.11, p.523-527, 1994.

LOW, P.; PANKSEPP, J.; REISS, D.; et al. 2012. The Cambridge Declaration on Consciousness. Disponível em: <http://fcmconference.org/img/CambridgeDeclar ationOnConsciousness.pdf> Acesso em 25/10/2013.

MELLOR, D. J.; GIBSON, T. J.; JOHNSON, C. $B$. A re-evaluation of the need to stun calves 
prior to slaughter by ventral-neck incision: an introductory review. New Zealand Veterinary Journal .v.57 n.2, p.74-76, 2009.

NHAT, P. H. Environmental performance improvement for small and medium-sized slaughterhouses in Vietnam environment. Development and Sustainability, v.8, p.251269, 2006.

NEVES, J.E.G. Influências de métodos de abate no bem-estar e na qualidade da carne de bovinos. Jaboticabal. 2008, 69f. Dissertação (Mestrado em Zootecnia) - Faculdade de Ciências Agrárias e Veterinária/Universidade Estadual Paulista Julio de Mesquita Filho".

Organização Internacional de Epizootias (OIE). Terrestrial animal code: Guidelines for the slaughter of animals. 2009. Disponível em: < http://www.oie.int/index.php?id=169\&L=0\&htmfil $\mathrm{e}=$ chapitre_1.7.5.htm $>$. Acesso em 30/9/2013.

OSBORNE, M. Horse slaughter conditions in Mexico explored by AAEP group. Journal of American Veterinary Medical Association, v.1, p.584-585, 2009.

RENNER, R. M. Fatores que afetam o comportamento, transporte, manejo e sacrifício de bovino. 87p. Monografia (Especialização) Universidade Federal do Rio Grande do Sul, Porto Alegre, 2005.

ROÇA, R.O. [2002]. Abate humanitário de bovinos. Disponível em:

<www.cpap.embrapa.br/agencia/congressovirtu $\mathrm{al} /$ pdf/portugues/> Acesso em: 22/11/2010.

SENG, P.M.; LAPORTE, R. Animal welfare: the role and perspective of the meat and livestock sector. Revue Scientifique et Technique (International Office Epizootics). , v. 24, n. 2, p.613-623, 2005.

VELARDE, A.; GISPERT, M.; DIESTRE, A.; MANTECA, X. Effect of electrical stunning on meat and carcass quality in lambs. Meat

Science. v. 63, p.35-38, 2003.

VIOLA, L.;CONSTANTINIDES, F.; Di NUNNO, C. et al. Suicide with a butcher's bolt. Journal of Forensic Sciences. v.49, p.595-597, 2004. 